\title{
Adoption of Hospitalist Care in Asia: Experiences From Singapore, Taiwan, Korea, and Japan
}

Yan Yan, PhD ${ }^{1}$, Toshio Naito, MD, PhD ${ }^{2 *}$, Nin-Chieh Hsu, MD, PhD³, Dong-Ho Shin, MD, MPH, DrPH ${ }^{4}$, Hyun-Jae Kang, MD, PhD ${ }^{5}$, Arpana R Vidyarthi, MD6, Susumu Tazuma, MD, PhD7, Jun Hayashi, MD, PhD ${ }^{8}$, Gautam A Deshpande, MD2,9

${ }^{1}$ Department of Palliative Medicine, Juntendo University Graduate School of Medicine, Tokyo, Japan; ${ }^{2}$ Department of General Medicine, Juntendo University Graduate School of Medicine, Tokyo, Japan; ${ }^{3}$ Division of Hospital Medicine, Department of Internal Medicine, National Taiwan University Hospital, Hsinchu City, Taiwan; ${ }^{2}$ Division of Integrated Medicine, Department of Internal Medicine, Severance Hospital, Yonsei University College of Medicine, Seoul, Korea; ${ }^{5}$ Department of Internal Medicine, College of Medicine, Seoul National University, Seoul, Korea; ${ }^{6}$ Division of Hospital Medicine, University of California, San Francisco, California (formerly Advanced Internal Medicine, National University Health System, Singapore); ${ }^{7}$ Department of General Internal Medicine, Hiroshima University Graduate School of Biomedical \& Health Sciences, Hiroshima, Japan; ${ }^{8}$ Haradoi Hospital, Fukuoka, Japan; ${ }^{9}$ Department of Internal Medicine, John A Burns School of Medicine, University of Hawaii, Honolulu, Hawaii.

ince its inception in the mid-1990s, the hospitalist model of care has enjoyed robust growth in the United States, increasing to around 20,000 providers by the end of its first decade., ${ }^{1,2}$ Since then, it has far outstripped early predictions of adoption, currently standing at more than 50,000 hospitalist providers. ${ }^{2}$ Although driven by numerous factors, including system-based management needs, provision of inpatient care for unassigned patients, and demands for improved patient safety and satisfaction, this meteoric growth has been driven largely by cost pressures particular to the US healthcare system. ${ }^{1,2}$ Nonetheless, the growing complexity of healthcare systems, substantial fiscal pressures, and increasing healthcare demands from aging populations are worldwide challenges to which countries outside North America also seek solutions. Countries that have initiated hospitalist care have localized adoption, evolving the model to meet their unique fiscal and system-based needs and patients' expectations.

While there has been keen interest in the hospitalist model in Asia, there has not yet been widespread adoption, despite numerous data demonstrating that this model is associated with lower length of stay (LOS), as well as lower costs and improved patient safety. ${ }^{3,4}$ This article explores hospitalist care adoption experiences in Singapore, Taiwan, Korea, and Japan, focusing on stakeholder demand for hospitalist-care, respective adoption, outcomes, and associated challenges to date.

\section{SINGAPORE}

\section{Stakeholder Demand for Hospitalist Care}

Historically in Singapore, family physicians provided primary care and internal medicine subspecialists provided inpatient care. ${ }^{5}$ Present-day trends, including an aging population, increasing rates of chronic diseases, and multisystem health

*Corresponding Author: Toshio Naito, MD, PhD; Email: naito@juntendo.ac.jp; Telephone: 03-5802-1190.

Published online first June 11, 2021.

Received: November 26, 2020; Revised: March 8, 2021; Accepted: March 19, 2021

๑ 2021 Society of Hospital Medicine DOI 10.12788/jhm.3621 issues, have stressed the historical model, leading to care fragmentation, long LOS (>9 days), and reduced patient satisfaction. ${ }^{5,6}$ Additionally, as $80 \%$ of hospital care is government funded, public hospitals are under pressure to reduce healthcare expenditures. ${ }^{5}$

\section{Adoption of Hospitalist Care, Outcomes, and Challenges Faced}

To meet patient needs and healthcare system challenges, the hospitalist model has evolved through several iterations in Singapore. The first model, implemented at Singapore General Hospital, utilized family physicians as hospitalists to coordinate inpatient care and integrate care between hospital and community settings. ${ }^{3,5}$ This model resulted in shorter LOS and reduced costs for patients cared for by family physician hospitalists. ${ }^{3}$ Despite these benefits, the family physician hospitalist model did not spread, partly due to biases favoring subspecialist care for hospitalized patients.?

The next iteration utilized general internal medicine (GIM) specialists. Traditionally, GIM specialists cared for a small number of low-acuity hospitalized patients. Recognizing the emerging need for holistic inpatient care, the Singapore Ministry of Health supported advances in generalist care, including a financial bonus and a revamped GIM training program. This spawned hospitalist-type models nationwide. At the National University Hospital (NUH), for example, GIM physicians were recruited to care for "specialty" patients in the acute medical unit and increase their ward coverage to include complex multimorbid patients. Additionally, NUH launched the enhanced complex care program, providing integrated inpatient and outpatient care to high-utilizing, complex patients. Overall, the NUH GIM division grew by $70 \%$ (faculty) and $60 \%$ (trainees) over 5 years. Currently, fueled by government enthusiasm for generalist care, hospitalist-type models are evident at newly minted hospitals across Singapore.

Although physicians act as hospitalists, the term hospitalist is not embraced in Singapore, thus limiting its potential to develop clinical- and system-improvement competencies and establish professional identity. This may be due to the strong UK-based cultural foundations and continued systemic bias favoring subspecialists. ${ }^{8}$ 


\section{TAIWAN}

\section{Stakeholder Demand for Hospitalist Care}

Under its national health insurance (NHI) system, Taiwan has relatively low copayments for medical services, with acute patients paying $10 \%$ of costs for a $\leq 30$-day hospitalization, causing demand for inpatient care to remain strong. ${ }^{4,9}$ The $\mathrm{NHI}$ system has also led to increased numbers of patients accessing care in emergency departments (EDs), where costs may be as low as US \$16 (NT \$450), causing long waits for evaluation and transfer to wards. ${ }^{9,10}$ There remains an insufficient number of hospital-based physicians to manage this high patient volume, a situation exacerbated by low reimbursements. ${ }^{4}$

\section{Adoption of Hospitalist Care, Outcomes, and Challenges Faced}

In order to address rising admissions, inefficient ED management, and physician shortages, a hospitalist-care program was first introduced in Taiwan in 2002, followed by the establishment of a hospitalist-run ward in National Taiwan University Hospital in 2009. ${ }^{11}$ Subsequent studies from Taiwan have found that hospitalist-run wards had lower admission costs, shorter LOS, and more do-not-resuscitate consent, and also had similar in-hospital mortality and readmission rates compared to specialist-run wards., ${ }^{4,12}$ Reflecting these successes, the Taiwan Association of Hospital Medicine (TAHM) was established in 2018, and since January 2021, the Ministry of Health and Welfare of Taiwan has mandated hospital medicine programs as an accreditation requirement for all medical centers, with a dual role of educating residents and providing inpatient care.

Despite growing opportunities, Taiwan has seen a modest increase in the number of hospitalists, rising from three in 2009 to around 300 by January 2021. An indistinct professional identity and career path are the main barriers. Given this, TAHM is trying to strengthen hospitalist professionalism by introducing both hard and soft skills, such as utilizing point-of-care ultrasonography and implementing the concepts of Choosing Wisely ${ }^{\circledR}$ and shared decision-making.

\section{KOREA}

\section{Stakeholder Demand for Hospitalist Care}

Korea has experienced a chronic physician shortage, with just 2.4 physicians per 1,000 people (World Bank, 2017), leading to significant physician burnout. Designed to protect trainee well-being, the 2015 Improvement of Training Conditions and Status of Medical Residents Act limited resident work hours while reducing internal medicine and general surgery training periods, further exacerbating physician shortages. ${ }^{13}$ In addition, Korea's current NHI system-including its healthcare insurance reimbursements scheme, established in 1989 when Korea's per capita gross domestic product was less than US $\$ 5,000$ - provides low reimbursements to healthcare providers. ${ }^{14}$ Along with increased attention to patient safety and healthcare-related consumer expectations, the hospitalist system in Korea aims to maintain improvements to residents' well-being, while increasing hospital revenue and meeting patient demand for improved services. ${ }^{14}$

\section{Adoption of Hospitalist Care, Outcomes, and Challenges Faced}

Along with the Ministry of Health and Welfare, the Korean Health Insurance Review and Assessment Service launched a hospitalist pilot program in general medicine and surgery in 2016. ${ }^{15}$ Services for hospitalist-managed inpatients are charged on a new schedule covered by the NHI system, including facility fees, which are charged per diem, and separate hospitalist fees. ${ }^{14}$ New hospital medicine programs are utilized, in part, to recruit new physicians to manage a large volume of inpatients. Previous studies found that these new hospitalist-care systems also improved patient safety, quality of care, and overall patient satisfaction, while being associated with shorter LOS and fewer unnecessary intensive care unit admissions. ${ }^{16,17}$ After a successful pilot, the revamped reimbursement system for hospitalist care officially started in January 2021.

Although Korea had only 250 registered hospitalists by August 2020, this is likely a substantial underestimate, as only hospital medicine teams with more than two hospitalists were allowed formal registration during the pilot period. Wider registration is currently underway for the new official reimbursement system.

\section{JAPAN}

\section{Stakeholder Demand for Hospitalist Care}

Hospitals in Japan are organized into highly compartmentalized subspecialties. Providing quality inpatient care to senior patients, who account for more than $28 \%$ of the population, and managing smooth transitions from hospital to long-termcare facilities remain challenging. In addition, given generous caps on maximum monthly out-of-pocket payments under its $\mathrm{NHI}$ system, LOS for Japanese hospitals are as long as 16.1 days. ${ }^{18}$ Nonetheless, given rising financial burdens associated with long-term care, hospitals are under government pressure to further shorten LOS and transition patients to local longterm-care facilities after treatment for acute symptoms.

\section{Adoption of Hospitalist Care, Outcomes, and Challenges Faced}

To meet these challenges, an increasing number of Japanese hospitals have established departments of general medicine to triage and manage patients with multiple comorbidities and to coordinate patient care across relevant specialties. The Japanese Society of Hospital General Medicine (JSHGM) was established in 2010, and currently has 1,890 members from 896 medical institutions. In 2018, general medicine was recognized by the Japanese Board of Medical Specialties as a formal specialty for certification. Currently, JSHGM is working with the Japan Primary Care Association and other organizations to establish a specialty certification system for hospitalist physicians and raise awareness of hospital medicine. A Japanese study of elderly patients with chronic aspiration pneumonia found that care by 
hospitalists resulted in shorter LOS and lower costs than specialist care..$^{19}$ Recently, hospitalists have played a central role in COVID-19 management, opening fever intake clinics and establishing collaborative guidelines with infectious disease experts and other specialists.

Yet, different from the prototypical hospitalist first defined by Wachter and Goldman, Japanese general medicine hospitalists continue to have substantial outpatient responsibilities, albeit in the hospital setting. Out of 81 university hospitals, 69 now have a department of hospital general medicine, though only 20 have inpatient services. ${ }^{20}$ In addition, a medical culture in which patients continue to see their surgery attendings long after surgery remains strong. Clear definitions regarding hospitalists' roles need to be established, while promoting changes toward inpatient care for both patients and subspecialists.

\section{DISCUSSION}

The four Asian countries reviewed here have all established universal access to healthcare, with Taiwan, Korea, and Japan having strong $\mathrm{NHI}$ systems and Singapore providing significant healthcare subsidies for those in need. Nonetheless, they also face similar challenges, including the growing complexity of healthcare systems, substantial fiscal pressures, increased healthcare demands caused by aging populations, and increased expectations regarding stakeholder well-being. As such, these countries share common driving forces that are propelling the adoption of hospitalist-care models, such as lack of a sufficient physician workforce on inpatient wards; need for extra resources to shorten ED wait times prior to inpatient admission; need for providing quality care to multimorbid senior patients across highly segmented hospital departments and coordinating medical services between hospitals and outpatient care facilities; and government pressure on cutting costs, especially by shortening inpatient LOS. Some common barriers among these Asian countries include unclear definitions of hospitalists' roles and degree of collaboration with subspecialty departments, and social and systemic biases favoring subspecialty care for inpatients.

The four Asian countries reviewed here have chosen to adopt the hospitalist model as a supplement to already established, specialty-driven inpatient care systems; as such, further comparative outcome studies focusing on cost, care quality, and patient safety and satisfaction are warranted to bolster professional hospitalist roles, further facilitate government/ policy-level support for hospital-care systems, and promote future training and certification systems appropriate to each country's unique healthcare system and medical culture. Similarly, evidence-driven educational outreach programs are warranted to facilitate patient understanding of the role of hospitalists in their care.

For countries interested in establishing hospital medicine programs, the adoption experiences in Singapore, Taiwan, Korea, and Japan provide valuable insights regarding how to establish hospitalist models to meet country-specific healthcare challenges while successfully functioning in the context of their unique medical-system frameworks.

Disclosures: Dr Deshpande reports receiving speaker fees from Daiichi-Sankyo unrelated to the submitted work. The other authors have nothing to disclose.

\section{References}

1. Wachter RM, Goldman L. The hospitalist movement 5 years later. JAMA 2002;287:487-494. https://doi.org/10.1001/jama.287.4.487

2. Wachter RM, Goldman L. Zero to 50,000 - the 20th anniversary of the hospitalist. N Engl J Med. 2016;375(11):1009-1011. https://doi.org/10.1056/ NEJMp1607958

3. Lee KH, Yang Y, Yang KS, Ong BC, Ng HS. Bringing generalists into the hospital: outcomes of a family medicine hospitalist model in Singapore. J Hosp Med. 2011;6:115-121. https://doi.org/10.1002/jhm.821

4. Shu CC, Lin JW, Lin YF, Hsu N-C, KoW-J. Evaluating the performance of a hospitalist system in Taiwan: a pioneer study for nationwide health insurance in Asia. J Hosp Med. 2011;6(7):378-382. https://doi.org/10.1002/jhm.896

5. Lee $\mathrm{KH}$. The hospitalist movement-a complex adaptive response to fragmentation of care in hospitals. Ann Acad Med Singap. 2008;37(2):145-150.

6. Ge L, Ya CW, Heng BH, Tan WS. Frailty and healthcare utilization across care settings among community-dwelling older adults in Singapore. BMC Geriatr. 2020;20(1):389. https://doi.org/10.1186/s12877-020-01800-8

7. Lee $\mathrm{KH}$. A historical perspective of the barriers to generalism. Aust Fam Physician. 2015;44(3):154-158.

8. Choo F. Alexandra Hospital provides patients with one-stop services under new care model. Updated December 14, 2018. Accessed March 26, 2021. https://www.straitstimes.com/singapore/health/alexandra-hospitalprovides-patients-with-one-stop-services-under-new-care-model

9. National Health Insurance Administration, Ministry of Health and Welfare, Taiwan. Medical services. Copayments. Updated December 28, 2020. Accessed March 26, 2021. https://www.nhi.gov.tw/English/Content_List. aspx?n=E5509C8FE29950EA\&topn=1D1ECC54F86E9050

10. Tsai JCH, Chen WY, Liang YW. Nonemergent emergency department visits under the National Health Insurance in Taiwan. Health Policy. 2011;100(2-3): 189-195. https://doi.org/10.1016/j.healthpol.2010.10.007

11. Taiwan Society of Hospital Medicine. The birth and growth of hospital medicine in Taiwan. Accessed March 26, 2021. https://www.hospitalist.org.tw/ about_25.htm

12. Hsu NC, Huang CC, Shu CC, Yang MC. Implementation of a seven-day hospitalist program to improve the outcomes of the weekend admission: a retrospective before-after study in Taiwan. PLoS One. 2018;13(3):e0194833. https://doi.org/10.1371/journal.pone.0194833

13. Ministry of Health and Welfare, Statutes of the Republic of Korea. Act on the Improvement of Training Conditions and Status of Medical Residents. Accessed March 26, 2021. https://elaw.klri.re.kr/eng_mobile/ viewer.do?hseq $=49563 \&$ type $=$ sogan\&key $=10$

14. Chae W, Park EC, Lee KY, et al. Development and evolution of hospital medicine in Korea. J Hosp Med. 2021;16(4):247-250. https://doi.org/10.12788/ jhm.3573

15. Oh SJ, Jung EJ. Prospects for the Korean model of the surgical hospitalist system. J Korean Med Assoc. 2020;63(5):236-239. https://doi.org/10.5124/ jkma.2020.63.5.236

16. Ohn JH, Kim NH, Kim ES, et al. An acute medical unit in a Korean tertiary care hospital reduces the length of stay and waiting time in the emergency department. J Korean Med Sci. 2017;32(16):1917-1920. https:// doi.org/10.3346/jkms.2017.32.12.1917

17. Lee JH, Kim AJ, Kyong TY, et al. Evaluating the outcome of multi-morbid patients cared for by hospitalists: a report of integrated medical model in Korea. J Korean Med Sci. 2019;34(25):e179. https://doi.org/10.3346/ jkms.2019.34.e179

18. OECD. Length of hospital stay. Accessed March 26, 2021. https://doi. org/10.1787/8dda6b7a-en

19. Hamada $O$, Tsutsumi $T$, Tsunemitsu $A$, et al. Impact of the hospitalist system in Japan on the quality of care and healthcare economics. Intern Med. 2019:58(23):3385-3391. https://doi.org/10.2169/internalmedicine.2872-19

20. Kawashima A. Report on general medicine's effects on specialists and other healthcare staff in the context of inclusive local medical system. Chapter in Japanese. Accessed March 26, 2021.https://soshin.pcmed-tsukuba.jp/education/report/pdf/05_004.pdf 